\title{
Movement related desynchronisation pattern preceding voluntary movement in untreated Parkinson's disease
}

\author{
L Defebvre, J L Bourriez, A Destée, J D Guieu
}

\begin{abstract}
Objective-To study planning of movement in Parkinson's disease.

Methods-The spatiotemporal pattern of movement related desynchronisation (MRD) preceding a self paced voluntary wrist flexion was compared between two groups of 10 untreated right and left hemiparkinsonian patients receiving no treatment and 10 control subjects. The MRD was computed in the 9 to $11 \mathrm{~Hz}$ frequency band from 11 source derivations covering the frontocentral, central, and parietocentral areas, during two successive left and right experimental conditions.
\end{abstract}

Results-In the two patient groups the desynchronisation appeared over the primary sensorimotor area contralateral to the affected side with a shorter latency (750 ms before movement onset for the right hemiparkinsonian group and $875 \mathrm{~ms}$ for the left hemparkinsonian group) than in the control group (1750 $\mathrm{ms})$, only when the movements were performed with the akinetic hand. For the non-affected hand, the same latency as in the control group was noted $(1750 \mathrm{~ms})$.

Conclusion-The delay of appearance of MRD in Parkinson's disease confirmed that the programming of movement is affected, thus partially explaining akinesia.

(F Neurol Neurosurg Psychiatry 1996;60:307-312)

Keywords: event related desynchronisation; Parkinson's disease; voluntary movement

Akinesia is one of the most characteristic features in Parkinson's disease. It corresponds to an inability to initiate movement and has been attributed to a perturbation in the programming of movement. ${ }^{1}$ The motor preparation period can be measured from the Bereitschaftspotential (BP), a slow cortical potential which occurs bilaterally 1.0 to 1.5 seconds before unilateral movement. ${ }^{2}$ During finger movements in patients with Parkinson's disease, the decrease of BP amplitude, which reflects in part the supplementary motor area (SMA) activity, was considered to result from a limitation of basal ganglia afferents. ${ }^{3}$ In the same way, before a stepping movement the absence of an increase in the BP might be interpreted as a perturbation in the preparation and initiation of walking. ${ }^{4}$ The results of
BP studies in Parkinson's disease are not uniform, as both clinical disability ${ }^{5}$ and levodopa treatment ${ }^{6}$ influence the size of the BP.

Movement related desynchronisation (MRD), which corresponds to a short amplitude attenuation of EEG intrinsic rhythms by a self paced voluntary movement, was first quantified in $1979 .{ }^{7}$ It can be used to study cortical activation during the planning of movement. In normal subjects MRD starts over the contralateral primary sensorimotor (PSM) area on average 1.7 seconds before movement, with a bilateral pattern after onset of movement. ${ }^{89}$ We recently performed simultaneous $\mathrm{BP}$ and $\mathrm{MRD}$ recordings during voluntary wrist flexion in patients with Parkinson's disease treated with levodopa. ${ }^{9} \mathrm{~A}$ delay in appearance of MRD over the PSM area was noted in the Parkinson's disease group compared with controls, mainly when the movements were executed with the more akinetic hand; by contrast there was no spatiotemporal difference for the BP between patients with Parkinson's disease and controls. Therefore MRD analysis might give more precise information about the period of motor preparation in Parkinson's disease.

The aim of this study was to compute MRD during self paced voluntary wrist movement in untreated hemiparkinsonian patients with left or right akinesia, (1) to determine which MRD pattern occurs at the first stage of the disease, when the patients are receiving no treatment, (2) to establish a possible correlation between the MRD spatiotemporal distribution, especially the latency, and the side of extrapyramidal symptoms.

\section{Patients and methods}

PATIENTS

Two groups of 10 right handed untreated patients with a diagnosis of idiopathic Parkinson's disease were studied. All the patients were recruited in the department of Neurology of Lille (France). They were rated Hoehn and $\mathrm{Yahr}^{10}$ grade I or II. In the right hemiparkinsonian (RHP) group (mean age $62 \cdot 2$ (SD 7.8) years), akinesia was localised on the right side in eight patients and with a pronounced predominance on this side in two patients; in the left hemiparkinsonian (LHP) group (mean age: $62 \cdot 9$, SD $8 \cdot 1$ years), akinesia was localised on the left side in seven patients and with a pronounced predominance on this side in three patients. The mean disease durations were 1.5 (SD 1.0) years (RHP group) and 1.9 (SD 1.2) years (LHP group). No 
patient had received antiparkinsonian treatment. Their motor disability was evaluated on the ?notor subscale of the unified Parkinson's discase rating scale (UPDRS). ${ }^{11}$ The mean scores were 23 (SD 15) (RHP group) and 18 (SD 14) (LHP group). The results were compared with those obtained in 10 right handed control subjects (mean age 64.5 (SD 5.8) years), participants in a community project for senior citizens.

The groups did not differ significantly in age, disease duration, and UPDRS score. The patients and normal subjects gave their informed consent to this study, which was approved by the local ethics committee.

\section{DATA ACQUISITION}

The subject sat in a reclining comfortable armchair with opened eyes, fixing vision on a point directly in front. Their forearms were placed on arm rests, each hand in a sheath, the wrists straightened, and the fingers in the straight resting position. The subject was asked to perform unilateral brisk self paced $45^{\circ}$ wrist flexion movements every 6 to 10 seconds, followed by a slow return to the resting position and to maintain the other hand at rest. The movement amplitude was limited by two copper abutments. A thin layer of copper situated on the sheath created an electrical contact with the first abutment and generated an impulse at the onset of movement. For each subject, two series of 100 movements were performed using the right and then the left hand; the first side was determined randomly. Electromyographic (EMG) activity was recorded (bandpass filters: $5 \mathrm{~Hz}-1 \mathrm{kHz}$ ) with bipolar $\mathrm{Ag}-\mathrm{AgCl}$ surface electrodes placed over the following muscles: left and right flexor carpi radialis and extensor digitorum communis to detect possible synkinesia of the resting hand. Signals were rectified and lower pass filtered to obtain the envelope of the signal. Latency between onset of agonist treated EMG, averaged over all trials, and movement onset, determined by the electrical contact, was also computed for each patient.

Electroencephalographic (EEG) activity was recorded from scalp electrodes with a common right mastoid reference, using 23

\footnotetext{
Figure 1 Position of scalp electrodes for EEG recording (common reference: $R E F)$. The 11 framed electrodes (source derivations) divided into derivations) divided into central, and parietocentral) are used to compute desynchronisation.
}

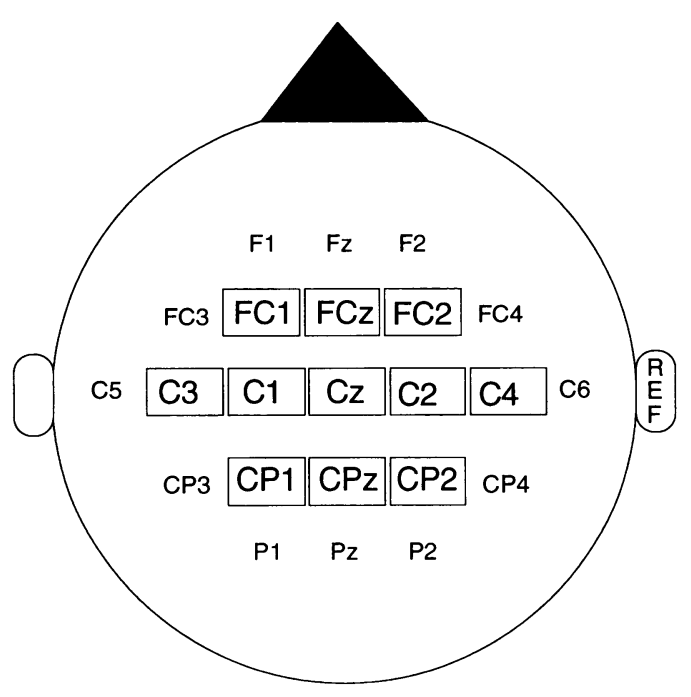

electrodes placed according to the international 10-20 system (fig 1). Bandpass filters were set at $0.5 \mathrm{~Hz}$ and $50 \mathrm{~Hz}$. The signals were sampled at $128 \mathrm{~Hz}$. The desynchronisation phenomenon was analysed from the continuous acquisition of 23 EEG channels 4 seconds before to 0.5 seconds after onset of movement. The MRD was computed using 11 source derivations ${ }^{1213}$ covering the frontocentral (FC1, FCz, FC2), central (C3, C1, Cz, $\mathrm{C} 2, \mathrm{C} 4)$, and parietocentral $(\mathrm{CP} 1, \mathrm{CPz}, \mathrm{CP} 2)$ areas. The quantification of $M R D$ is based on the averaging technique. Each 4.5 second artefact free period of EEG was digitally bandpass filtered in the 9 to $11 \mathrm{~Hz}$ frequency band. The samples were squared and averaged over all trials to obtain the mean power evolution. To obtain a normalised measure of MRD the power decrease was expressed as a percentage of a reference power value computed within the time interval from 3.5 seconds to $2.5 \mathrm{sec}$ onds before wrist flexion. To reduce the variance, temporal resolution was reduced to obtain one power value every $125 \mathrm{~ms}$. Further details about data processing are reported elsewhere. ${ }^{89}$

Afterwards we compared for each group the percentages of desynchronisation obtained at one moment for one derivation to the zero value, using a Wilcoxon test for matched samples (level of significance $1 \%$ ). When this test was significant, the mean $\%$ value obtained in the group at this moment and for this localisation was retained to draw a spatiotemporal map.

Two repeated measures analysis of variance (ANOVA; controls/untreated RHP; controls/ untreated LHP) were also performed to evaluate the effects of group (control, RHP, LHP), side of movement (right, left), electrode location (frontocentral, left central, vertex, right central, parietocentral), time (17 epochs of $125 \mathrm{~ms}$ from 2 seconds before to $125 \mathrm{~ms}$ after movement onset) on the \% MRD values. A $5 \%$ level of significance was adopted.

\section{Results}

Figures 2, 3, and 4 show the spatiotemporal maps in the $9-11 \mathrm{~Hz}$ frequency band. Time in seconds is expressed on the horizontal axis (time interval of $125 \mathrm{~ms}$ ), 2 seconds before and 0.5 seconds after the onset of movement (time 0 ) determined by the electrical contact. Mean latency of agonist EMG onset is also specified on each map (the table summarises the values for each group). In the control group, with right as well as left wrist flexion, desynchronisation began $1750 \mathrm{~ms}$ before onset of movement. The MRD was localised over the contralateral PSM (C2 and C4 for the left flexion, $\mathrm{C} 1$ and $\mathrm{C} 3$ for the right flexion) with a bilateral central pattern, which appeared when the movement was executed. Desynchronisation was also found over the parietocentral zone for the two experimental conditions with a contralateral predominance before movement and a bilateral pattern after onset of movement, whereas no desynchronisation over the frontocentral area was noted. 
Figure 2 Spatiotemporal maps of MRD (9-11 Hz) in the control group (10 subjects). Right wrist flexion (top) and left wrist flexion (bottom). Time in seconds is expressed on the horizontal axis (time interval of $125 \mathrm{~ms})$, two seconds before and 0.5 seconds after the movement onset (time 0). Agonist EMG onset is indicated by a vertical arrow (mean value for each group is specified in the table). Locations of source derivations are noted in three groups on the vertical axis: frontocentral (FC1, $F C z, F C 2)$, central (C3, $C 1, C z, C 2, C 4)$, and parietocentral (CP1, CPz, $C P 2)$. The mean $\% M R D$ is expressed with grey colour coding. As stressed in the text, only the significant values of $\%$ $M R D(P<0 \cdot 01)$ were used to draw the map.

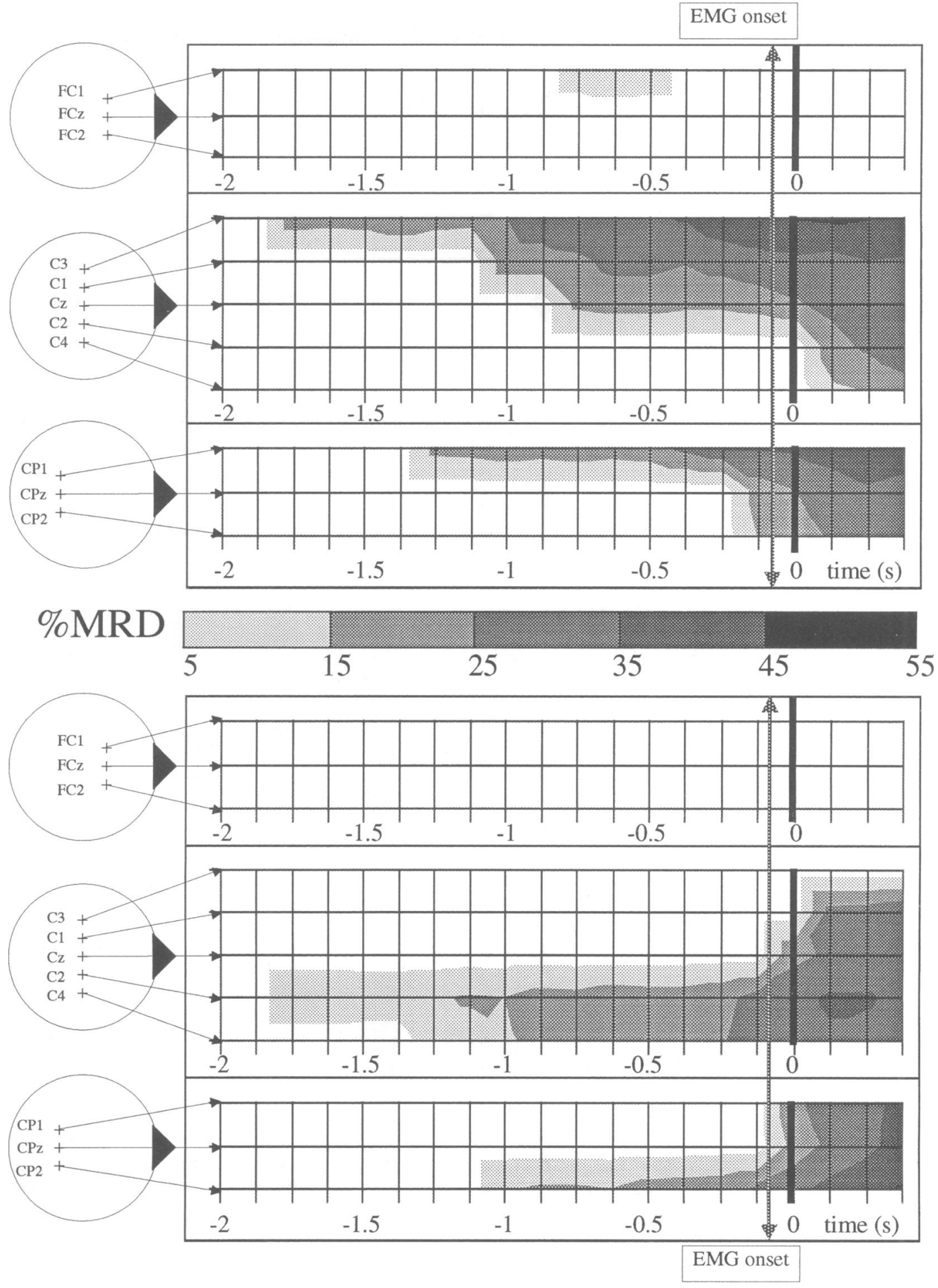

For the two untreated groups the contralateral and ipsilateral distributions differed from the control group. As in controls MRD appeared also over the contralateral PSM and

Mean (SD) latency between agonist EMG onset and movement onset (time 0) defined by the first electrical contact, in the control and the untreated RHP and LHP groups

\begin{tabular}{lll}
\hline & Right flexion $(\mathrm{ms})$ & Left flexion $(\mathrm{ms})$ \\
\hline Controls & \multicolumn{1}{c}{$88(27)$} & $\mathbf{8 4}(14)$ \\
Untreated & $160(60)$ & $134(50)$ \\
RHP & $(\mathrm{z}=-2 \cdot 76, \mathrm{P}<0.01)$ & $(\mathrm{z}=-2 \cdot 41, \mathrm{P}<0.05)$ \\
Untreated & $126(22)$ & $138(43)$ \\
LHP & $(\mathrm{z}=-2 \cdot 54, \mathrm{P}<0.05)$ & $(\mathrm{z}=-2 \cdot 84, \mathrm{P}<0.01)$ \\
\hline
\end{tabular}

Significant differences are between each Parkinson's diseas group and controls (Mann-Whitney test). the parietocentral area but the latency was shorter when the movement was executed with the akinetic hand (RHP: $750 \mathrm{~ms}$, LHP: 875 $\mathrm{ms})$ and normal $(1750 \mathrm{~ms})$ with the other hand. Distribution of MRD over the ipsilateral sensorimotor area appeared earlier, 250 to 750 ms before movement. The MRD was also noted over the frontocentral area in the two experimental conditions in the LHP group.

Analysis of variance, showing significant main effect of time (RHP: $F(16,576)=$ 15.54, P < 0.01; LHP: $F(16,576)=12.95$, $\mathrm{P}<0.01$ ), electrode position (LHP: $F(4,576)$ $=9.94$, P<0.01; LHP: $F(4,576)=4.74$, $P<0.01$ ), and flexion by electrode interaction (RHP: $F(4,576)=9.26, \mathrm{P}<0.01$; LHP: $F$ 
Figure 3 Spatiotemporal maps of MRD $(9-11 \mathrm{~Hz})$ in the untreated RHP group (10 patients). Same legend as fig 2.
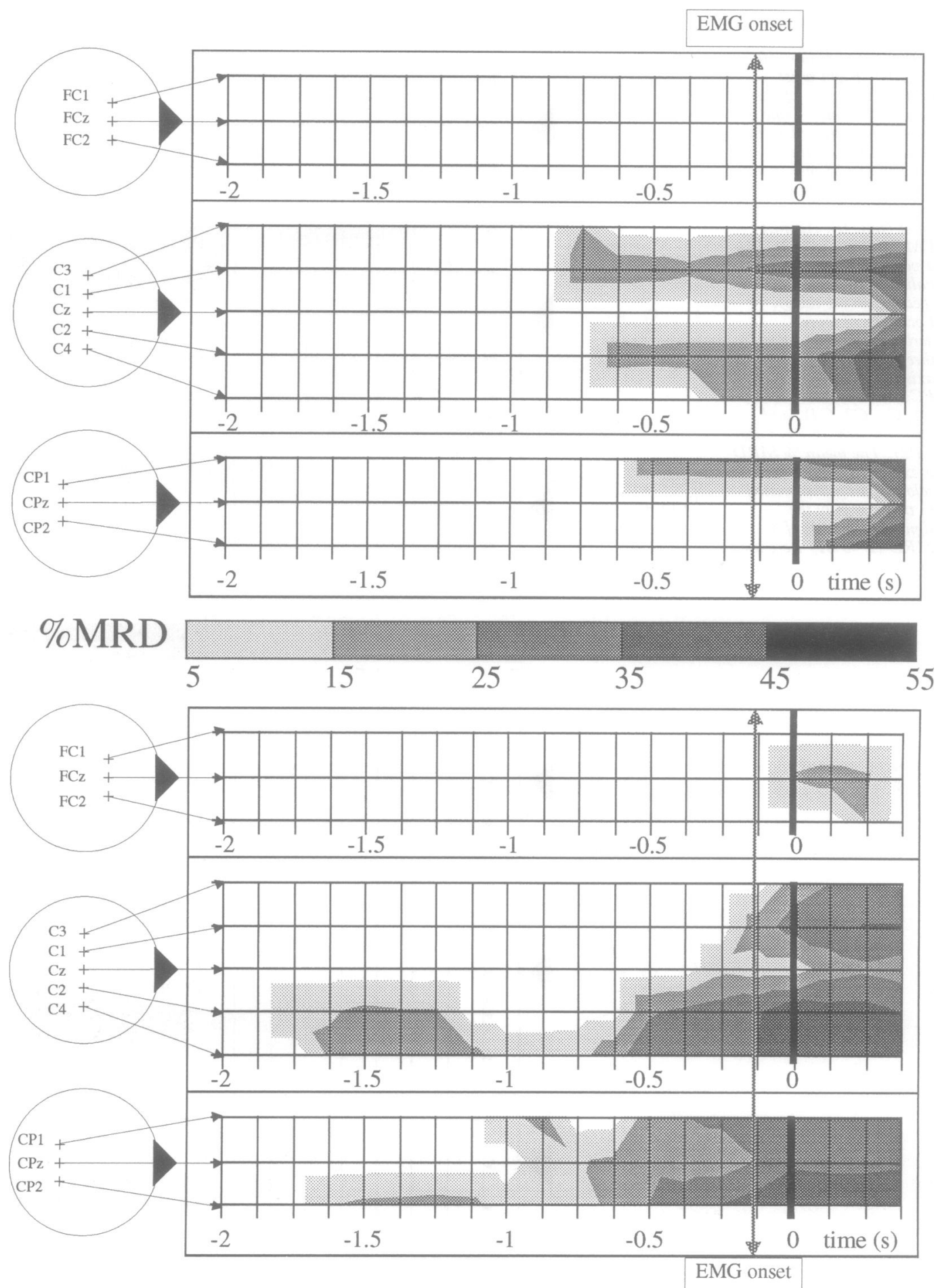

$(4,576)=16 \cdot 2, P<0.01)$ confirmed these patterns. A significant flexion by group interaction (RHP: $F(1,576)=4.96, \mathrm{P}<0.05$; LHP: $F(1,576)=6.51, P<0.05)$ suggested that the side of movement affected MRD results for both untreated groups, compared with controls.

For each experimental condition and for each untreated group, no synkinesia on the other forearm was recorded with EMG. The table gives the mean latency of agonist EMG onset. For each group there was no difference between the right and left movements, whereas for the two untreated Parkinson's disease groups mean latencies of agonist EMG were significantly different from controls.

\section{Discussion}

During a motor process, MRD can be considered as an indicator of cortical activation, with a high time resolution and a specific spatial representation over the cortical areas, obtained by using source derivations. ${ }^{8}$ These features make MRD suitable for studying the motor preparation period preceding a voluntary movement. According to the relations between scalp electrodes and cortical areas, the PSM cortex underlies the central derivations (C1, C2 and C3, C4). ${ }^{14}$ In the control group MRD appeared over the contralateral PSM area, almost two seconds before movement, with a bilateral pattern after onset of movement (fig 2). The later bilateralisation of MRD over the 
Figure 4 Spatiotemporal maps of MRD $(9-11 \mathrm{~Hz})$ in the untreated LHP group (10 patients). Same legend as fig 2.
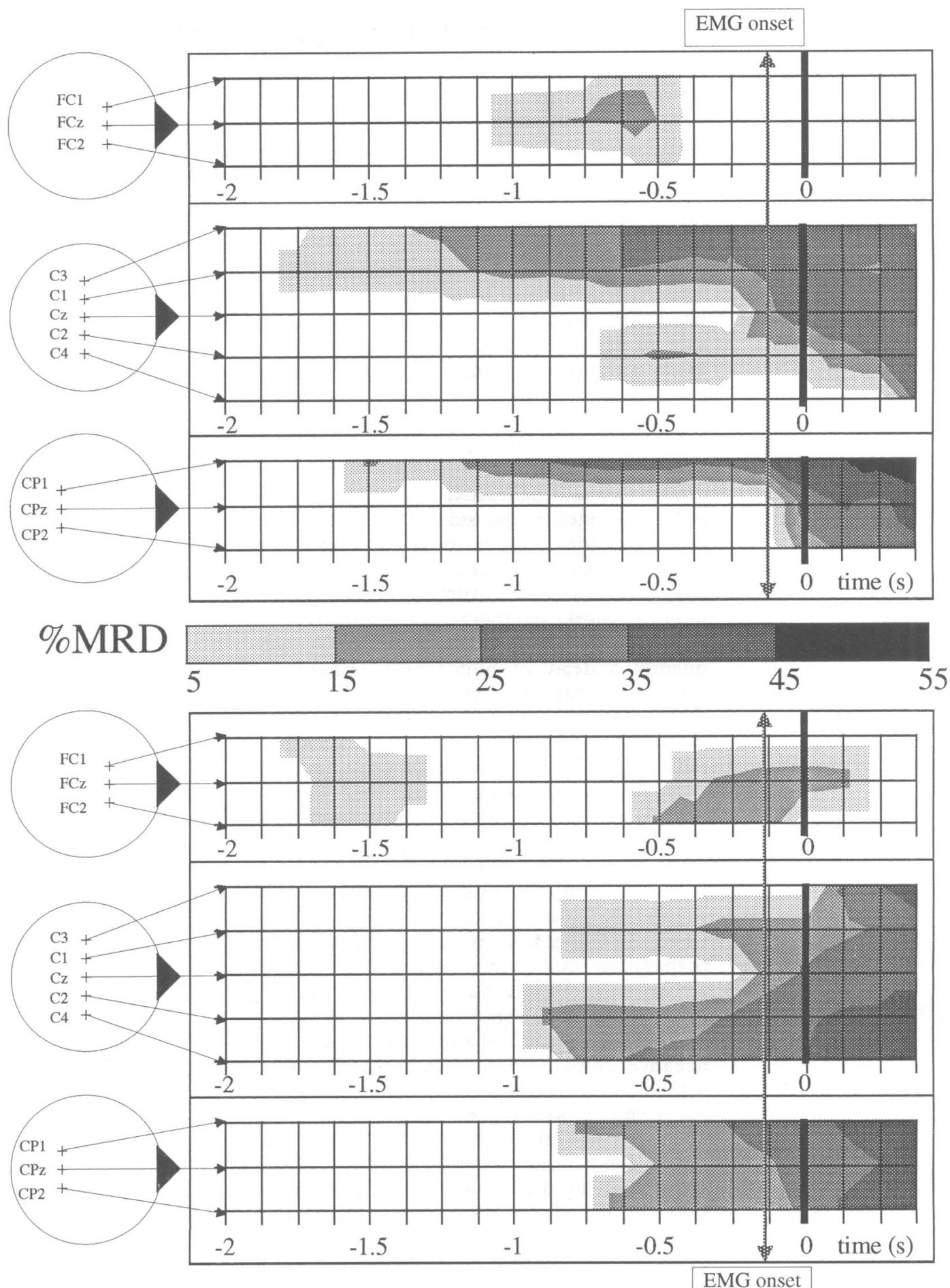

central area, seen after the wrist flexion, confirms the double activation of the primary motor cortex during a unilateral movement, as has already been suggested by studies of regional cerebral blood flow. ${ }^{15} 16$

By contrast, the untreated parkinsonian patients showed different spatial and temporal MRD distribution. In both groups desynchronisation appeared over the contralateral PSM area with a shorter latency (figs 3 and 4), but only when the movements were performed with the akinetic side (RHP: $750 \mathrm{~ms}$, LHP: $875 \mathrm{~ms}$ ). The planning of movement is a complex process that involves not only the PSM area but also the SMA, premotor area, basal ganglia, thalamus nuclei, and cerebellum. ${ }^{17}$
The SMA receives some afferents of basal ganglia via the ventrolateral thalamic nuclei and sends information to the primary cortex..$^{18}$ In Parkinson's disease this cortical structure dysfunction resulting from a deficiency of nigrostriatal dopamine. We suggested that this deactivation process can explain that MRD latency, which reflects onset of cortical activity during the motor preparation period, was reduced in both Parkinson's disease groups when the movements were executed with the akinetic hand. The delay in appearance of MRD in Parkinson's disease could reflect the time required to reach a threshold level of cortical activity for execution of movement. receives less input from the basal ganglia, their 
Moreover, if the agonist EMG onset was retained to define the movement onset, the reduction in MRD latency noted in the two untreated groups appeared still more pronounced, whereas the difference between EMG onset and movement onset, determined by the electrical contact, was more important in both groups than in controls (table). For the other experimental condition (non-affected hand) the same latency as in the control group was noted (1750 ms before movement onset). Thus MRD latency seems to be well correlated with clinical symptoms, especially on the side of akinesia. These results are in agreement with those obtained in a group of 10 patients with Parkinson's disease (mean disease duration: $6 \cdot 8$ (SD 4.1) years, Hoehn and Yahr grade I-III), receiving levodopa. ${ }^{9}$ Latency of MRD was reduced for the two conditions (left and right wrist flexions) mainly for right movements, the side more affected by extrapyramidal symptoms. We consider that the difference in latency seen in the untreated Parkinson's disease groups reflects a lower motor cortical excitability, as has already been proved in human parkinsonism ${ }^{19}$ and consequently a delay of motor programming, in agreement with the hypothesis of Marsden ${ }^{1}$ that in Parkinson's disease the programming of movement would be affected, at least partially explaining the mechanism of akinesia.

In the two untreated Parkinson's disease groups, an ipsilateral pattern of the desynchronisation over the central and parietocentral areas was noted earlier than in the control group for both conditions. This pattern could suggest that other cortical areas might be activated to compensate for dysfunction of motor preparation and increase the level of cortical activity required to execute a movement. This interpretation has also been suggested by Dick et al in a BP study ${ }^{20}$ : the reduction of the first negative slope (NS1) reflects poor cortical activation of the system involving SMA and basal ganglia, whereas the augmented negativity in the late phase of the BP (NS2) could reflect compensatory activity of other cortical areas involving the dorsolateral primary motor area and visual sensory systems. This earlier ipsilateral distribution could also be related to imitation synkinesia of the other hand, but this hypothesis is excluded, because no synkinesia on the other forearm was recorded with EMG.

Our study indicates that MRD analysis is a useful and simple method to observe the changes in cortical activation during the motor preparation period and to measure some aspects of motor programming. The use of source derivations gives a clear advantage for the topographical analysis of MRD. A good correlation was obtained between the latency of MRD over the PSM area in both hemiparkinsonian groups and the side of akinesia. Further studies after a few months of levodopa treatment in untreated patients with Parkinson's disease should specify the influence of levodopa on the evolution of the MRD pattern, and may have important clinical implications for the use of MRD as an electrophysiological marker of clinical symptoms in Parkinson's disease.

1 Marsden CD. The mysterious motor function of the basal ganglia: the Robert Wartenberg lecture. Neurology 1982;32:514-39.

2 Shibasaki H, Barrett G, Halliday E, Halliday AM. Components of the movement-related cortical potential and their scalp topography. Electroencephalogr Clin Neurophysiol 1980;49:213-26.

3 Deecke L, Englitz HG, Kornhuber HH, Schimtt G. Cerebral potentials preceding voluntary movements in parents poth bilats preceding voluntary movements in In: Desmedt JE, ed. Attention, voluntary contraction and In: Desmedt JE, ed. Attention, voluntary contraction and event-related cerebral potentials.

4 Vidailhet $M$, Stocchi F, Rothwell JC, et al. The Bereitschaftspotential preceding simple foot movement and initiation of gait in Parkinson's disease. Neurology 1993;43:1784-8

5 Fève AP, Bathien N, Rondot P. Evolution des potentiels corticaux liés au mouvement chez les patients parkinsoniens, avant et après traitement par la lévodopa. Neurophysiol Clin 1991;21:105-19.

6 Dick JPR, Cantello $R$, Buruma $O$, et al. The Bereischaftspotential, L-dopa and Parkinson's disease. Electroencephalogr Clin Neurophysiol 1987;66:263-74.

7 Pfurtscheller G, Aranibar A. Evaluation of event-related desynchronization (ERD) preceding and following voluntary self-paced movement. Electroencephalogr Clin untary self-paced movement.

8 Pfurtscheller G, Berghold A. Patterns of cortical activation during planning of voluntary movement. Electroduring planning of voluntary moveme

9 Defebvre L, Bourriez JL, Dujardin K, Derambure P, Destée A, Guieu JD. Spatiotemporal study of Bereitschaftspotential and event-related desynchronization during voluntary movement in Parkinson's disease. Brain Topography 1994;6:237-43.

10 Hoehn MM, Yahr MD. Parkinsonism: onset, progression and mortality. Neurology 1967;17:427-42.

11 Fahn S, Elton RL and members of the UPDRS development committee. Unified idiopathic Parkinson's disease rating scale. In: Fahn S, Marsden CD, Calne D, rating scald In. Fann ease. Vol 2. Florham Park, NJ: MacMillan Healthcare ease. Vol 2. Florham Park, NJ:

12 Hjorth B. An on-line transformation of EEG scalp potentials into orthogonal source derivation. Electroencephalogr tials into orthogonal source derivation
Clin Neurophysiol 1975;39:526-30.

13 Pfurtscheller G. Mapping of event-related desynchronization and type of derivation. Electroencephalogr Clin Neurophysiol 1988;70:190-3.

14 Homan RW, Herman J, Purdy P. Cerebral location of 10-20 system electrode placement. Electroencephalogr Clin Neurophysiol 1987;66:376-82.

15 Roland PE. Cortical organization of voluntary behavior in man. Human Neurobiology 1985;4:155-67.

16 Shibasaki $\mathrm{H}$, Sadato N, Lyshkow $\mathrm{H}$ et al. Both primary motor cortex and supplementary motor area play an important role in complex finger movement. Brain important role in

17 Goldberg G. Supplementary motor area stucture and function: review and hypotheses. Behavioral Brain Science tion: review and

18 Jurgens U. The efferent and afferent connections of the supplementary motor area. Brain Res 1984;300:63-81.

19 Davey NJ, Dick JPR, Ellaway PH, Maskill DW. Raised motor cortical threshold associated with bradykinesia as reavealed by transcranial magnetic stimulation in norma man and Parkinson's disease. F Physiol 1991;438:35P.

20 Dick JPR, Rothwell JC, Day BL, et al. The Bereischaftspotential is abnormal in Parkinson's disease. Brain 1989;112:233-44. 